\section{La regulación de precios de medicamentos en Colombia: el caso del tenecteplase}

\section{Regulation of drug prices in Colombia: the case of tenecteplase}

\section{Sr. Editor:}

La regulación de precios de medicamentos ha sido una de las estrategias del Ministerio de Salud y Protección Social para enfrentar la crisis financiera del sistema ${ }^{1}$. El impacto esperado de esta medida se debe reflejar tanto en los medicamentos de más alto costo como en aquellas condiciones que, como el infarto agudo de miocardio, tienen incidencia alta. Se estima que para el año 2011, murieron 29.373 colombianos a causa de un infarto agudo de miocardio ${ }^{2}$. El acceso a la trombólisis con tenecteplase (TNK), intervención recomendada por las guías internacionales ${ }^{3,4}$, así como por la Sociedad Colombiana de Cardiología ${ }^{5}$, es aún limitado. Según la base de datos de SISMED, en 2014 se vendieron en Colombia 2.115 unidades de TNK, a un costo total de $\$ 7.973 .720 .696$ pesos (US\$3.982.877 al cambio medio de 2014). En un estudio de costo-efectividad del TNK realizado en 2013 con costos de ese mismo año, que consideraba solamente costos directos para el sistema de salud con un horizonte temporal de toda la vida del paciente, se encontró que el costo de emplear TNK en lugar de la terapia estándar de entonces (que empleaba estreptoquinasa), supondría un costo por año de vida ajustado por calidad (AVAC) de $\$ 22.927 .336$ (valor que era superior al PIB de ese año, pero inferior a 3 veces ese valor) lo que lo dejaba en la categoría de probablemente costo-efectivo ${ }^{6}$.

El 4 de febrero de 2015, mediante Circular 03, el precio máximo del TNK fue establecido en $\$ 45.467$ pesos por miligramo, equivalente a $\$ 2.273 .339$ la ampolla. Al aplicar este precio al mismo modelo de 2013, el costo por AVAC ganado se reduciría a $\$ 20.659 .472$, algo más cercano, pero todavía superior, al PIB per cápita. Esta reducción del precio, de un $39,7 \%$, podría también interpretarse de tal manera que, si en el año siguiente a la fecha de la Circular se emplearan las mismas unidades de TNK en el país, el ahorro para el sistema de salud sería de $\$ 3.165 .609 .769$ pesos, o si se gastara la misma suma que se pagó en 2014 se alcanzarían a tratar 1.392 pacientes más, suponiendo que cada paciente requiere una sola dosis de TNK.

La regulación de precios de medicamentos es tan solo una de las múltiples intervenciones que requiere el sistema de salud colombiano, pero es sin duda una medida necesaria para la sostenibilidad del mismo.

\section{Bibliografía}

1. Franco-Giraldo A. La última reforma del sistema general de seguridad social en salud Colombiano. Rev Salud Pública. 2012;14:865-77.

2. Ministerio de Salud y Protección Social. Colombia enfrenta epidemia de enfermedades cardiovasculares y diabetes. [consultado 20 Jul 2015]. Disponible en: http://www.minsalud.gov.co/ Paginas/Colombia-enfrenta-epidemia-de-enfermedadescardiovasculares-y-diabetes.aspx

3. O'Gara PT, Kushner FG, Ascheim DD, Casey DE Jr, Chung MK, de Lemos JA, et al. 2013 ACCF/AHA guideline for the management of ST-elevation myocardial infarction: a report of the American College of Cardiology Foundation/American Heart Association Task Force on Practice Guidelines. Circulation. 2013;127:362-425.

4. National Institute for Health and Care Excellence. Acute management of myocardial infarction with ST-segment elevation: summary of NICE guidance. BMJ. 2013;347:406.

5. Sociedad Colombiana de Cardiología y Cirugía Cardiovascular. Guía colombiana de cardiología: síndrome coronario agudo con elevación del segmento ST. Rev Col Cardiol. 2010;17:121-275.

6. Moreno A. Análisis de costo-efectividad de la trombólisis con tenecteplasa vs. estreptoquinasa en el manejo del infarto agudo de miocardio con supradesnivel del ST en Colombia. [Trabajo de Grado para Maestría]. Bogotá: Pontifica Universidad Javeriana; 2013.

Diego Rosselli*, Atilio Moreno, Andrea Martínez y Pieralessandro Lasalvia

Departamento de Epidemiología Clínica y Bioestadística, Pontificia Universidad Javeriana Bogotá, Colombia

* Autor para correspondencia.

Correo electrónico: diego@tinieblo.com (D. Rosselli). 